\title{
HSP70 silencing aggravates apoptosis induced by hypoxia/reoxygenation in vitro
}

\author{
CONGYING ZHAI ${ }^{1,2}$, JILING LV ${ }^{2}$, KEKE WANG ${ }^{3}$, QINGSHU LI ${ }^{1}$ and YAN QU ${ }^{1}$ \\ ${ }^{1}$ Intensive Care Unit, Affiliated Qingdao Municipal Hospital of Qingdao University, Qingdao, Shandong 266071; \\ ${ }^{2}$ Department of Respiratory Medicine, Zibo First Hospital, Zibo, Shandong 255200; ${ }^{3}$ Department of Emergency, \\ Affiliated Qingdao Municipal Hospital of Qingdao University, Qingdao, Shandong 266071, P.R. China
}

Received June 8, 2018; Accepted February 28, 2019

DOI: $10.3892 /$ etm.2019.7697

\begin{abstract}
Lung ischemia-reperfusion can cause acute lung injury, which is closely associated with apoptosis. Heat shock protein 70 (HSP70) is an anti-apoptotic protein that promotes cell survival under a variety of different stress conditions. However, the role and mechanism of HSP70 in lung ischemia-reperfusion injury is yet to be fully elucidated. In the present study, an in vitro hypoxia/reoxygenation model of A549 cells was established to simulate lung ischemia-reperfusion and HSP70 was silenced by transfecting A549 cells with an shRNA sequence targeting HSP70. Western blotting, reverse transcription-quantitative polymerase chain reaction, Cell Counting kit- 8 and flow cytometry were used to detect protein levels, RNA expression, cell activity and apoptosis. The results revealed that silencing HSP70 reduced cell viability, aggravated apoptosis, increased lactate dehydrogenase levels and induced a G2/M blockade in a hypoxia-reoxygenation A549 cell model. Furthermore, silencing HSP70 decreased the phosphorylation levels of protein kinase B (AKT) and extracellular signal-regulated kinase (ERK); however, the total AKT and ERK levels did not change significantly. Pretreating A549 cells with the AKT pathway inhibitor, LY294002 and the ERK pathway inhibitor, U0216 led to a decrease in HSP70 expression. These results indicate that silencing HSP70 may aggravate apoptosis in hypoxia-reoxygenation cell models, potentially via the mitogen-activated protein kinase/ERK and phosphoinositide 3-kinase/AKT signaling pathways.
\end{abstract}

Correspondence to: Dr Yan Qu, Intensive Care Unit, Affiliated Qingdao Municipal Hospital of Qingdao University, 1 Jiaozhou Road, Qingdao, Shandong 266071, P.R. China

E-mail: qdquyan@aliyun.com

Key words: heat shock protein 70, apoptosis, hypoxia, reoxygenation, phosphoinositide 3-kinase, protein kinase $B$, mitogen-activated protein kinase, extracellular signal-regulated kinase

\section{Introduction}

Lung ischemia-reperfusion injury (LIRI) is characterized by non-specific alveolar damage, lung edema, pulmonary hypertension and hypoxemia (1), and has been implicated in several clinical conditions including cardiopulmonary resuscitation, lung transplantation and pulmonary embolism (2-4). LIRI has a relatively complicated pathological mechanism, which serves an important role in apoptosis. Previous studies have revealed that cell apoptosis is associated with an increased risk of LIRI, indicating that anti-apoptosis may be a potential target to treat lung ischemic disease $(5,6)$.

Heat shock protein 70 (HSP70) is highly conserved and is activated by stress stimulation (7). The main function of HSP70 is to participate in the regulation of intracellular protein folding and to protect cells from stress damage $(8,9)$. HSP70 has been demonstrated to affect anti-apoptotic functions (10). A previous study have revealed that the overexpression of HSP70 effectively protects alveolar epithelial cells against apoptosis and antagonizes the effects of LIRI (11). Furthermore, the overexpression of HSP70 also prevents proteins from misfolding and aggregating $(12,13)$. The overexpression of HSP70 also attenuates ischemia-reperfusion injury (IRI) induced by lung transplantation (14). In addition, several studies have reported that the overexpression of HSP70 reduces heart (15) and brain (16) IRI. However, to the best of our knowledge, the mechanism that underlies the protective effect of HSP70 on LIRI has not yet been elucidated.

The B-cell lymphoma ( $\mathrm{Bcl})-2$ family of proteins is comprised of pro-apoptotic proteins, including $\mathrm{BH} 3$ interacting domain death agonist and Bcl-2-associated X (Bax), and anti-apoptotic proteins including Bcl-2 and Bcl-extra large. Jayanthi et al (17) demonstrated that Bcl-2 and Bax regulates apoptosis and that apoptosis is dependent on the ratio of $\mathrm{Bcl}-2 / \mathrm{Bax}$; the lower the $\mathrm{Bcl}-2 / \mathrm{Bax}$ ratio, the more severe the level of apoptosis.

The caspase family are enzymatic proteins that serve key roles in the signal transduction of apoptosis (18). One of the family members, caspase-3, is the core effector and main executor of apoptosis (19) that is positively correlated with apoptosis. Mitogen-activated protein kinase (MAPK)/extracellular signal-regulated kinase (ERK) 
and phosphoinositide 3-kinase (PI3K)/protein kinase $\mathrm{B}$ (AKT) are important pathways for maintaining cell survival $(20,21)$. The two pathways are activated during lung ischemia $(22,23)$, indicating that they may be involved in the pathological process of pulmonary ischemia.

The present study assessed the protective effect of HSP70 on LIRI as well as its underlying mechanism. Levels of Bcl-2, Bax, caspase-3 and lactate dehydrogenase (LDH), and cell cycle and apoptotic rate were analyzed to assess the protective effects of HSP70. In addition, the expression of phosphorylated (p)-ERK and p-AKT were determined. Finally, the involvement of the MAPK/ERK and PI3K/AKT signaling pathways were evaluated using their respective inhibitors.

\section{Materials and methods}

Reagents and antibodies. Dulbecco's modified Eagle's medium (DMEM) and fetal bovine serum (FBS) were purchased from Hyclone (GE Healthcare Life Sciences, Logan, UT, USA). The anti-HSP70 antibody was purchased from Abcam (Cambridge, UK). The anti-Caspase-3, anti-Bcl-2, anti-Bax, anti-ERK, anti-p-ERK, anti-AKT, anti-p-AKT, anti-GAPDH and anti- $\beta$-Actin antibodies, as well as LY294002 (the PI3K/AKT inhibitor) and U0126 (the MAPK/ERK inhibitor) were all purchased from Cell Signaling Technology, Inc. (Danvers, MA, USA).

Cell culture. A549 cells (Type Culture Collection of the Chinese Academy of Sciences, Shanghai, China) were cultured and passaged in DMEM containing 10\% FBS at $37^{\circ} \mathrm{C}$ in a humidified atmosphere with $5 \% \mathrm{CO}_{2}$. Cells were divided into three groups: The recombinant lentiviral infection group (lentivirus mediated knockdown of the HSP70 gene), the lentivirus control group (empty lentiviral vector) and the non-infection group.

Infection. A549 cells were transfected with a lentiviral overexpression vector $\left(1 \times 10^{7} \mathrm{TU} / \mathrm{ml}\right.$; Shanghai GeneChem Co., Ltd., Shanghai, China) that expressed HSP70 and green fluorescent protein (GFP). The lentiviral vector containing an shRNA sequence (5'-GGACGAGTTTGA GCACAAG-3'), which targeted HSP70 or the lentiviral vector backbone according to the manufacturer's protocol. Briefly, $4 \times 10^{4}$ cells/well were seeded into 24 -well plate and incubated at $37^{\circ} \mathrm{C}$ in $5 \% \mathrm{CO}_{2}$ overnight. According to the pretesting, $20 \mu 1$ lentivirus liquid, $80 \mu 1$ Enhanced Infection Solution, $50 \mu \mathrm{g} / \mathrm{ml}$ polybrene (both Shanghai GeneChem Co., Ltd.) and $800 \mu 1$ DMEM were added directly onto the cell monolayer. After incubating at $37^{\circ} \mathrm{C}$ for $12 \mathrm{~h}$, the normal DMEM was added. After $48 \mathrm{~h}$ of lentivirus transfection, GFP-transfected cells and total cells were counted by two researchers independently under a fluorescence microscope (each researcher randomly selected 5-7 fields of vision at a magnification of $x 400$ and counted more than 700 cells). The average infection rate was calculated as follows: Infection rate $=$ green fluorescent cells/total cells x 100\%. Stable cells were screened by puromycin (Invitrogen; Thermo Fisher Scientific, Inc., Waltham, MA, USA) for culture and passaged.
Hypoxia/reoxygenation cell model. A549 cells were cultured in DMEM without glucose or serum and placed in a hypoxic environment at $37^{\circ} \mathrm{C}$ for $2,4,6,8$ and $10 \mathrm{~h}$. Hypoxic conditions were established using an Anaeropack (Mitsubishi Gas Chemical Company, Inc., Tokyo, Japan), which contained sodium ascorbate as the principal ingredient to absorb oxygen and generate the same volume of carbon dioxide via oxidative degradation in a sealed container (24). Subsequently, glucose-free DMEM (Beijing Solarbio Science \& Technology Co., Ltd., Beijing, China) was replaced with normal DMEM and the cells were cultured in a reoxygenated environment with $21 \% \mathrm{O}_{2}$ and $5 \% \mathrm{CO}_{2}$ for $24 \mathrm{~h}$ at $37^{\circ} \mathrm{C}$. U0126 or LY294002 were dissolved in dimethyl sulfoxide (DMSO). The stock solutions were prepared with minimal exposure to air and light, and stored at $-20^{\circ} \mathrm{C}$ for $1 \mathrm{~h}$ prior to hypoxia/reoxygenation treatment. Then $10 \mu \mathrm{M}$ U0126 or $50 \mu \mathrm{M}$ LY294002 from the stock solutions were added to the uninfected A549 cells (the U0126 group) before hypoxia/reoxygenation. DMSO was added to the non-infection, lentivirus control and lentiviral infection groups.

Cell viability assay. A549 cells were seeded at a density of $8 \times 10^{3}$ in 96 -well plates and incubated at $37^{\circ} \mathrm{C}$ for $24 \mathrm{~h}$. Subsequently, cells were exposed to hypoxic conditions for 2, 4, 6, 8 and $10 \mathrm{~h}$ and reoxygenized for a further $24 \mathrm{~h}$. Finally, $10 \mu \mathrm{l}$ of Cell Counting kit-8 (CCK-8; Wuhan Boster Biological Technology, Ltd., Wuhan, China) solution was added to each well according to the manufactures protocol and incubated at $37^{\circ} \mathrm{C}$ for $1 \mathrm{~h}$. The absorbance of each well was then measured at $450 \mathrm{~nm}$ using a microplate reader.

Apoptosis analysis. Following $6 \mathrm{~h}$ of hypoxia and $24 \mathrm{~h}$ of reoxygenation, the three groups of cells were collected with $0.25 \%$ trypsin. An Annexin V-allophycocyanin/propidium iodide (PI) staining kit (eBioscience; Thermo Fisher Scientific, Inc.) was utilized for the detection of apoptosis according to the manufacturer's protocol. Following washing with PBS and binding buffer, the cells were resuspended in Annexin V binding buffer $\left(1 \times 10^{6}\right.$ cells $\left./ \mathrm{ml}\right)$. Subsequently, $5 \mu \mathrm{l}$ of fluorochrome-conjugated Annexin V was added to $100 \mu \mathrm{l}$ of cell suspension and incubated for $10-15 \mathrm{~min}$ at room temperature. Cells were then further washed with binding buffer and resuspended in $200 \mu \mathrm{l}$ of binding buffer.

A total of $5 \mu 1$ of the PI staining solution was added to the cell suspension, which was then analyzed using a FACStar Plus flow cytometer (BD Biosciences, Franklin Lakes, NJ, USA). Data analysis was performed with BD Accuri C6 software (BD Biosciences).

Cell cycle analysis. A PI Flow Cytometry kit (Abcam) was used to determine cell cycle status with a flow cytometer. The three groups of cells were cultured under hypoxic conditions for $6 \mathrm{~h}$ and then reoxygenated for $24 \mathrm{~h}$ prior to being harvested into a single cell suspension. According to the manufacturer's protocol, the cells were fixed in $66 \%$ ethanol at $4^{\circ} \mathrm{C}$ for $2 \mathrm{~h}$, rehydration in PBS and stained with PI + RNase from the PI Flow Cytometry kit at $4^{\circ} \mathrm{C}$ for $30 \mathrm{~min}$. Finally, the fluorescence intensity of PI was measured at $488 \mathrm{nM}$ on FL2 using a flow cytometer. Data 
analysis was performed with FlowJo VX10 software (FlowJo LLC, Ashland, OR, USA).

Reverse transcription-quantitative polymerase chain reaction (RT-qPCR) detection. Total RNA was extracted using TRIzol (Invitrogen; Thermo Fisher Scientific, Inc.) and reverse transcribed into cDNA with PrimeScript RT reagent kit (Takara Bio, Inc., Otsu, Japan). Briefly, $1 \mu \mathrm{g}$ total RNA of each sample was used for each reaction in a $20 \mu \mathrm{l}$ reaction mixture. After removing genomic DNA using gDNA Eraser (Takara Bio, Inc.), cDNA was synthesized at $37^{\circ} \mathrm{C}$ for 15 min. qPCR was performed using cDNA as a template with the FastStart Essential DNA Green Master kit (Roche Diagnostics, Basel, Switzerland) and $\beta$-actin as a reference. The reaction conditions included an initial pre-denaturation at $95^{\circ} \mathrm{C}$ for $10 \mathrm{~min}$, followed by 45 cycles of $95^{\circ} \mathrm{C}$ for $15 \mathrm{sec}$, $60^{\circ} \mathrm{C}$ for $20 \mathrm{sec}$ and $72^{\circ} \mathrm{C}$ for $15 \mathrm{sec}$. After the termination of the PCR, the production was analyzed by the LightCycler96 System (Roche Diagnostics, GmbH, Mannheim, Germany) automatically. The primers used were as follows: $\beta$-actin forward, 5'-GATGAGATTGGCATGGCTTT-3' and reverse, 5'-CACCTTCACCGTTCCAGTTT-3'; HSP70 forward, 5'-CAAGAAGAAGGTGCTGGACA-3' and reverse, 5'-GTA CAGTCCGCTGATGATGG-3'. The relative level of HSP70 mRNA was calculated using the $2^{-\Delta \Delta C q}$ method (25).

Western blot analysis. Following hypoxia-reoxygenation treatment, the cells in each group were collected. Cells were lysed with RIPA lysis buffer containing phenylmethylsulfonyl fluoride (both Beyotime Institute of Biotechnology, Shanghai, China) within $20 \mathrm{~min}$. The concentration was determined with BCA Protein Assay kit (Beijing Transgen Biotech Co., Ltd., Beijing, China) and adjusted to a concentration of $40 \mu \mathrm{g}$ per well. The protein sample and loading buffer were mixed in proportion and denatured at $95^{\circ} \mathrm{C}$ for $5 \mathrm{~min}$, and then subjected to SDS-PAGE (10\% stacking gel and 5\% separating gel) with the conditions, $80 \mathrm{~V}$ for $40 \mathrm{~min}$ and $110 \mathrm{~V}$ for $80 \mathrm{~min}$. Following electrophoresis, the proteins were transferred to a polyvinylidene difluroide membrane. The membrane was then blocked for $2 \mathrm{~h}$ with $5 \%$ skim milk solution at room temperature, washed three times with TBST solution and incubated with the following primary antibodies overnight at $4^{\circ} \mathrm{C}$ : Anti-HSP70 (cat. no. 47455; 1:1,000), anti-AKT (cat. no. 4691; 1:1,000), anti-p-AKT (cat. no. 4060; 1:2,000), anti-ERK (cat. no. 4695; 1:1,000), anti-p-ERK (cat. no. 4370; 1:2,000), anti-caspase-3 (cat. no. 9662; 1:1,000), anti-Bcl-2 (cat. no. 4223; 1:1,000), anti-Bax (cat. no. 5023; 1:1,000), anti-GADPH (cat. no. 5174; $1: 1,000)$ and anti- $\beta$-actin (cat. no. 4970; 1:1,000). Samples were then incubated with horseradish peroxidase-conjugated rabbit anti-mouse (cat. no. 6728; 1:2,000; Abcam) and goat anti-rabbit (cat. no. 7074; 1:2,000; Cell Signaling Technology, Inc.) immunoglobulin $\mathrm{G}$ secondary antibodies for $2 \mathrm{~h}$ at room temperature. Developing in darkroom with West Pico ECL Kit (Millipore, Billerica, MA, USA). Quantity One 4.4 software (Bio-Rad Laboratories, Inc., Hercules, CA, USA) was used to analyze the integrated optical density of each band.

LDH assay. The LDH assay kit (cat. no C0017) was purchased from Beyotime Institute of Biotechnology. All measurements were collected in accordance with the manufacturer's protocol.
Statistical analysis. Data were analyzed using SPSS 23.0 statistical software (IBM Corp., Armonk, NY, USA). All results were presented as the mean \pm standard deviation. One-way analysis of variance followed by a Least Significant Difference test was performed to compare differences between the means in more than two groups. $\mathrm{P}<0.05$ was considered to indicate a statistically significant difference.

\section{Results}

Lentiviral transfection inhibits the expression of HSP70. At $48 \mathrm{~h}$ post-transfection, A549 cells appeared to be polygon-shaped, plump and interconnected under the fluorescence microscope (Fig. 1). The infection rate of A549 cells, was $\sim 80 \%$. Compared with the lentivirus control group and the non-infection group, a significant decrease was observed in the mRNA (Fig. 2A) and protein (Fig. 2B and C) levels of HSP70 in the lentivirus infection group $(\mathrm{P}<0.05)$.

HSP70 silencing decreases A549 cell viability. In order to select the effective duration of hypoxia, the cell viability under hypoxic conditions was measured. The results of the CCK- 8 assay revealed that, at $6 \mathrm{~h}, \mathrm{~A} 549$ cells had the highest marked viability compared with other time points (Fig. 3). In addition, the viability of the lentivirus infection group was significantly lower than that of the lentivirus control and non-infection groups at all time points $(\mathrm{P}<0.05)$.

HSP70 silencing increases the number of apoptotic A549 cells following hypoxia and reoxygenation. Following $6 \mathrm{~h}$ of hypoxia and $24 \mathrm{~h}$ of reoxygenation, the rate of apoptosis in the lentiviral infection group was significantly higher when compared with the other two groups $(\mathrm{P}<0.05$; Fig. 4).

Hsp70 silencing increases caspase-3 expression and decreases the ratio of $\mathrm{Bcl}-2 / \mathrm{Bax}$. The results of western blotting revealed that the expression of caspase- 3 in the lentiviral infection group was increased by $\sim 80 \%$ when compared with the non-infection and lentivirus control groups $(\mathrm{P}<0.05$; Fig. 5A and $\mathrm{B})$. Furthermore, the ratio of Bcl-2/Bax was decreased by $70.1 \%$ when compared with the lentivirus control group ( $\mathrm{P}<0.05$; Fig. 5A and $\mathrm{C})$.

HSP70 silencing causes G2/M arrest. The ratio of G2 and M phase cells in the lentiviral infection group was significantly higher than that of the non-infection and lentivirus control groups $(\mathrm{P}<0.05$; Fig. 6).

HSP70 silencing promotes cellular $\mathrm{LDH}$ release. $\mathrm{LDH}$ levels in the lentiviral infection group were significantly higher when compared with the non-infection and lentivirus control groups $(\mathrm{P}<0.05$; Fig. 7$)$. These results indicate that HSP70 silencing promotes the release of LDH.

Influence of HSP70 on the MAPK/ERK signaling pathway. Cells were exposed to hypoxia for $6 \mathrm{~h}$ and reoxygenation for $24 \mathrm{~h}$ with or without $10 \mu \mathrm{M} \mathrm{U} 0126$ pretreatment. The results revealed that treatment with $10 \mu \mathrm{M}$ U0126 effectively inhibited ERK phosphorylation and decreased HSP70 expression compared with the non-infection group 

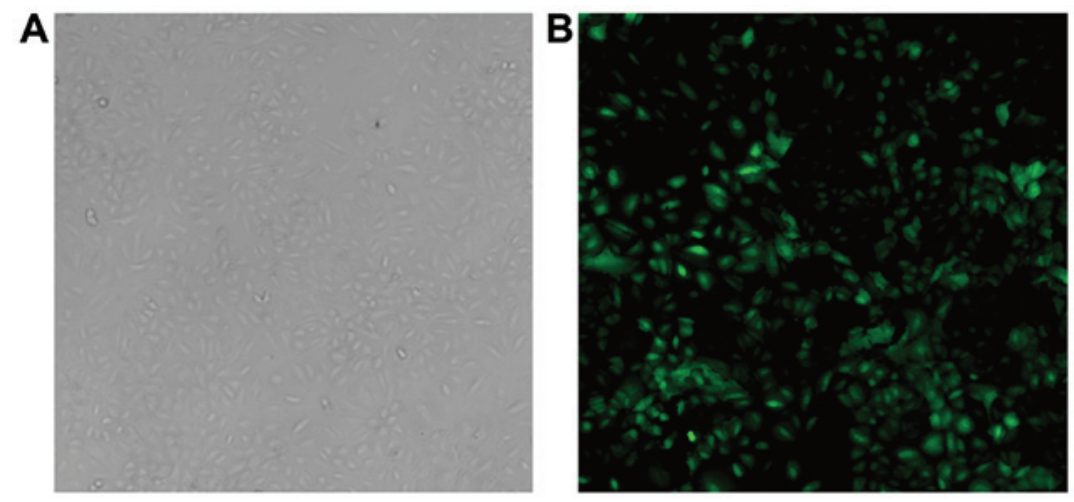

Figure 1. Lentivirus transfection efficiency detected via fluorescence microscopy. A549 cells were transfected with the recombinant lentivirus containing the shRNA sequence of HSP70 (magnification, x100). Green fluorescence was observed in successfully transfected A549 cells. A549 Cells after $48 \mathrm{~h}$ of infection under (A) white and (B) fluorescent light. The infection efficiency was $~ 80 \%$. shRNA, short hairpin RNA; HSP70, heat shock protein 70.

A

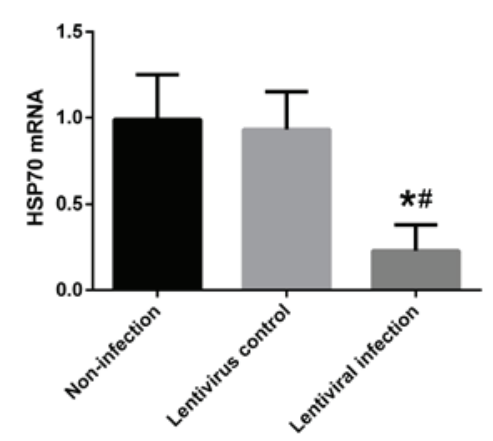

B

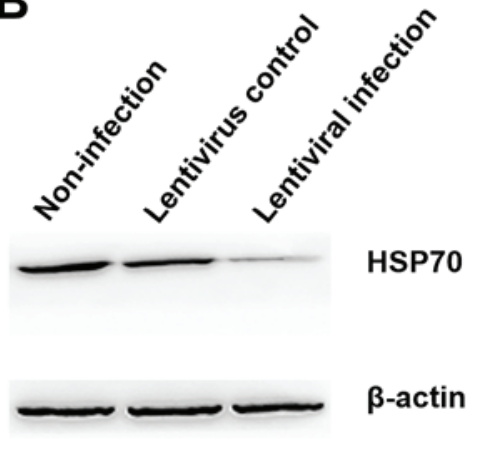

C

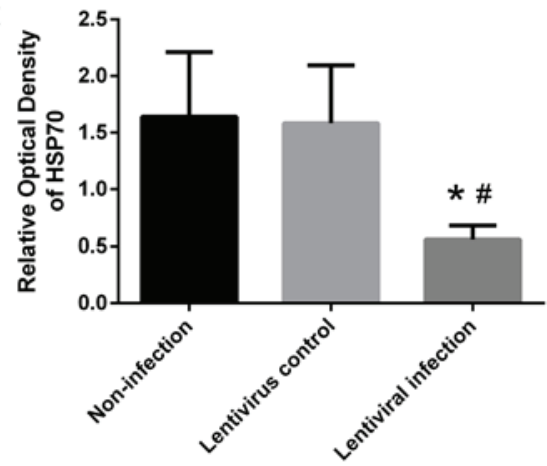

Figure 2. Expression of HSP70 mRNA and protein in A549 cells. The (A) mRNA and (B) protein expression of HSP70 was determined via reverse transcription-quantitative polymerase chain reaction and western blotting. (C) The result of western blotting was quantified three times. ${ }^{*}<<0.05$ vs. the non-infection group; ${ }^{\mathrm{P}}<0.05$ vs. the lentivirus control group. HSP70, heat shock protein 70 .

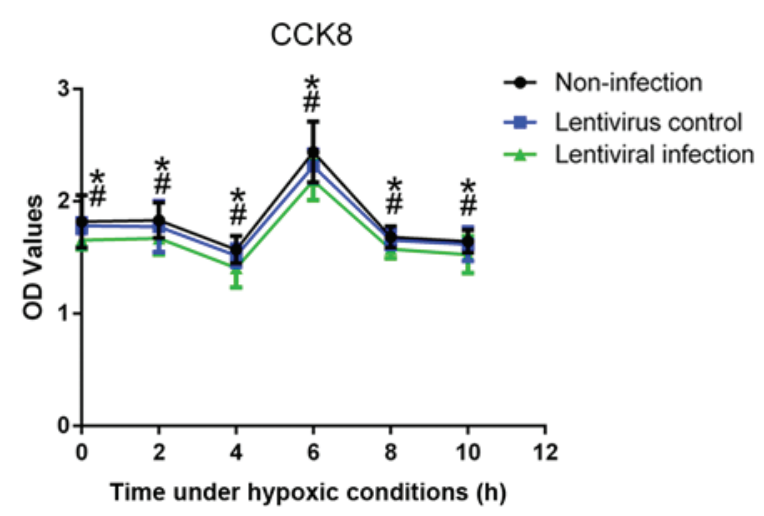

Figure 3. A549 cell viability as determined via a cell CCK-8 assay following different durations of hypoxia and reoxygenation for $24 \mathrm{~h}$. The viability of the lentivirus infection group was significantly lower than that of the lentivirus control and non-infection groups at all time points. Data are presented as the mean \pm standard deviation. ${ }^{*} \mathrm{P}<0.05$ vs. the non-infection group; ${ }^{\#} \mathrm{P}<0.05$ vs. the lentivirus control group. $\mathrm{CCK}$, cell counting kit.

(Fig. 8A and B). In addition, HSP70 silencing decreased the level of p-ERK compared with the non-infection group $(\mathrm{P}<0.05$; Fig. 8A and $\mathrm{C})$.
Influence of HSP70 on the PI3K/AKT signaling pathway. Cells were exposed to hypoxia for $6 \mathrm{~h}$ and reoxygenation for $24 \mathrm{~h}$ with or without $50 \mu \mathrm{M}$ LY294002 pretreatment. The results revealed that treatment with $50 \mu \mathrm{M}$ LY294002 effectively inhibited AKT phosphorylation and decreased HSP70 expression compared with the non-infection group $(\mathrm{P}<0.05$; Fig. 9A and B). In addition, HSP70 silencing decreased the level of $\mathrm{p}$-AKT compared with the non-infection group $(\mathrm{P}<0.05$; Fig. 9A and $\mathrm{C})$. Treatment with $10 \mu \mathrm{M}$ U0126 and $50 \mu \mathrm{M}$ LY294002 also reduced the mRNA level of HSP70 compared with the non-infection group (Fig. 10).

\section{Discussion}

LIRI causes severe lung injury and involves a series of pathological processes including oxidative stress, inflammation and apoptosis $(26,27)$. Apoptosis is an important method of cell death following LIRI (28). HSP70, a member of the most conserved family of HSPs, usually serves as a regulator and signaling molecule in certain cellular processes, such as cell damage resistance and repair, and protects cells from various stress-induced injuries, including apoptosis, oxidative stress and ischemic-reperfusion injury (29). The high expression of HSP72 in alveolar epithelial cells induced by dimethylarsinic acid has been demonstrated to inhibit 
A

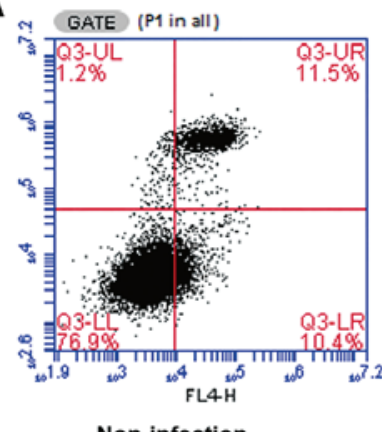

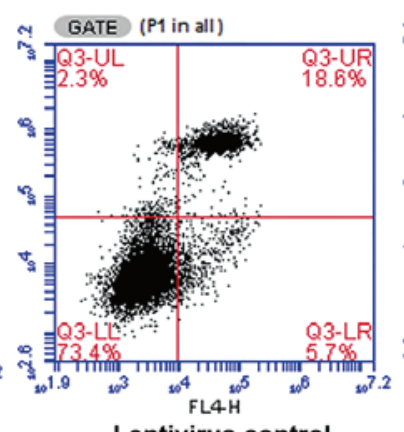

Lentivirus control
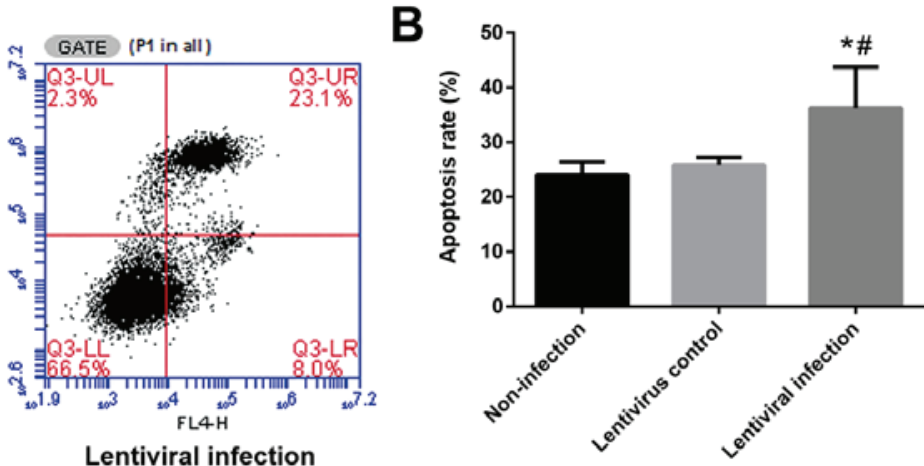

Figure 4. A549 cells apoptosis following hypoxia/reoxygenation. Following A549 cell exposure to hypoxia for $6 \mathrm{~h}$ and then reoxygenation for 24 h, the apoptosis rate of cells was measured via flow cytometry with Annexin V-allophycocyanin/propidium iodide. Representative (A) flow cytometry results and $(\mathrm{B})$ apoptosis ratio. Data are presented as the mean \pm standard deviation. ${ }^{*} \mathrm{P}<0.05$ vs. the non-infection group; ${ }^{\#} \mathrm{P}<0.05$ vs. the lentivirus control group.

A

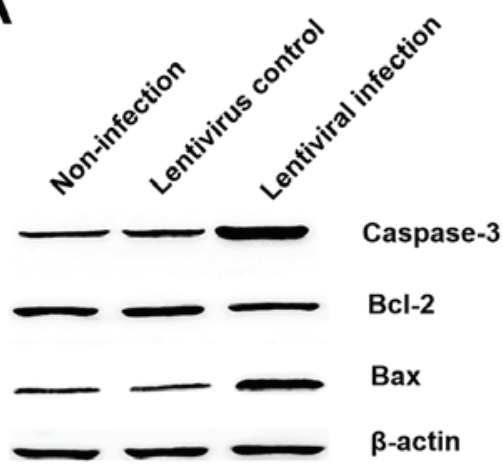

B

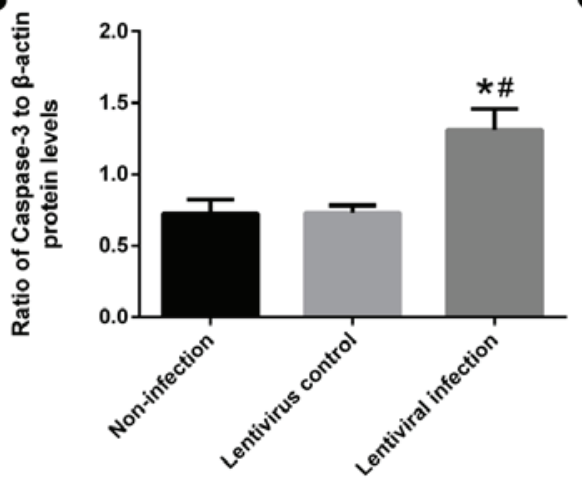

C

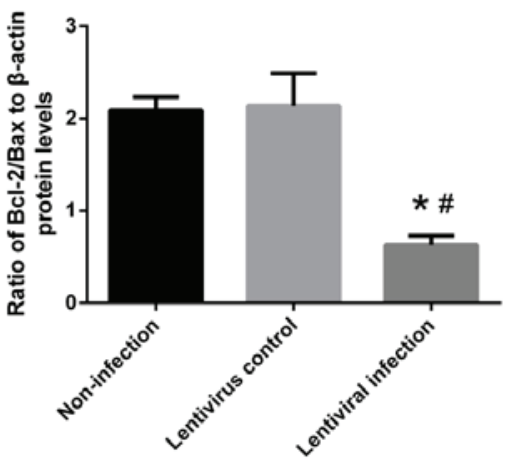

Figure 5. Effect of HSP70 silencing on the protein expression of caspase-3, Bcl-2 and Bax. (A) Western blotting was performed to assess (B) caspase-3 and (C) Bcl-2 and Bax protein levels. Data are presented as the mean \pm standard deviation ${ }^{*} \mathrm{P}<0.05$ vs. the non-infection group; ${ }^{*} \mathrm{P}<0.05$ vs. the lentivirus control group. HSP70, heat shock protein 70; Bcl-2, B-cell lymphoma 2; Bax, Bcl-2-associated X.

cell apoptosis (30). Lipopolysaccharide-induced acute lung injury can also be protected against via an increased HSP70 expression (31). In addition, the overexpression of HSP70 exhibits a protective effect on LIRI (11). As aforementioned, HSP70 is an anti-apoptotic protein that provides protection against hypoxic/reoxygenation injuries (32). In the current study, HSP70 silenced A549 cells exhibited a marked reduction in Bcl-2 and a marked increase of Bax and caspase-3. Furthermore, apoptotic rates were increased, indicating an increase in cellular apoptosis. LDH is an important indicator of cell injury and as such was assessed in the present study. The results of LDH analysis revealed that HSP70 silencing increased the release of $\mathrm{LDH}$ in cells, indicating an increase in cell injury. The results of the current study therefore revealed that HSP70 silencing aggravated cell injury and apoptosis in a model of hypoxia-reoxygenation. Thus, it was inferred that the cytoprotective effect of HSP70 on LIRI may be associated with anti-apoptosis and the mitigation of cell injury. Interestingly, the authors of the current study found that the proliferation of A549 cells increased following $6 \mathrm{~h}$ of hypoxia. This may be because a short period of hypoxia stimulates cell proliferation, but long-term hypoxia reduces cell activity $(33,34)$.
All phases, including $\mathrm{G} 2 / \mathrm{M}$, have detection points for cell cycle regulation. DNA damage caused by various factors can lead to G2/M phase arrest, which is closely associated with apoptosis (35). The results of the present study revealed that HSP70 silencing resulted in G2/M arrest in A549 cells following hypoxia/reoxygenation, reducing cell proliferation. It is possible that apoptosis induced by HSP70 silencing may therefore be associated with cell cycle arrest.

ERK is a serine/threonine protein kinase and belongs to the MAPK family (36). It is an important transduction protein that transmits mitogen signals within the body (37). AKT is a direct target gene of PI3K. Activating PI3K can phosphorylate Akt, which causes downstream phosphorylation cascades and interactions between target proteins, thus regulating cell survival and apoptosis (38). It has been demonstrated that ERK (39) and AKT (40) enhance cell survival by stimulating phosphorylation in downstream targets, thereby serving a protective role in the lung. Furthermore, the anti-apoptotic effect of HSP70 is closely associated with the ERK (41) and AKT (42) pathways. To gain further insight into the mechanism by which HSP70 protects against hypoxia/reoxygenation-induced apoptosis, the present study assessed the role of the MAPK/ERK and 
A

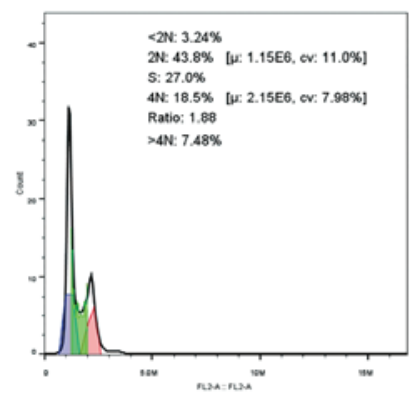

Non-infection

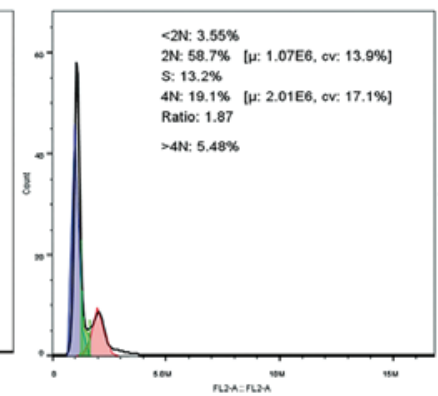

Lentivirus control

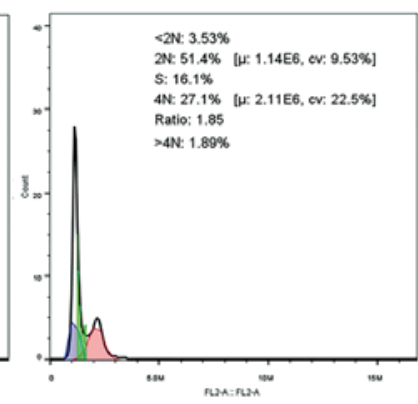

Lentiviral infection
B

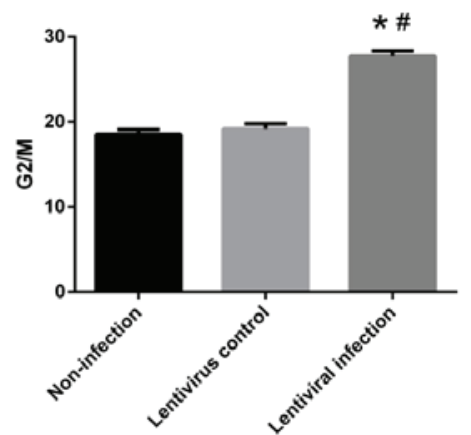

Figure 6. HSP70 silencing arrests G2/M phase. Cell cycle distribution was detected using a flow cytometer following A549 cell exposure to hypoxia for 6 h and reoxygenation for $24 \mathrm{~h}$. (A) Flow cytometry results and (B) the ratio of $\mathrm{G} 2 / \mathrm{M}$ phase cells are presented. ${ }^{*} \mathrm{P}<0.05$ vs. the non-infection group; ${ }^{\#} \mathrm{P}<0.05$ vs. lentivirus control group. HSP70, heat shock protein 70; 2N, G1 phase; 4N, G2/M phase.

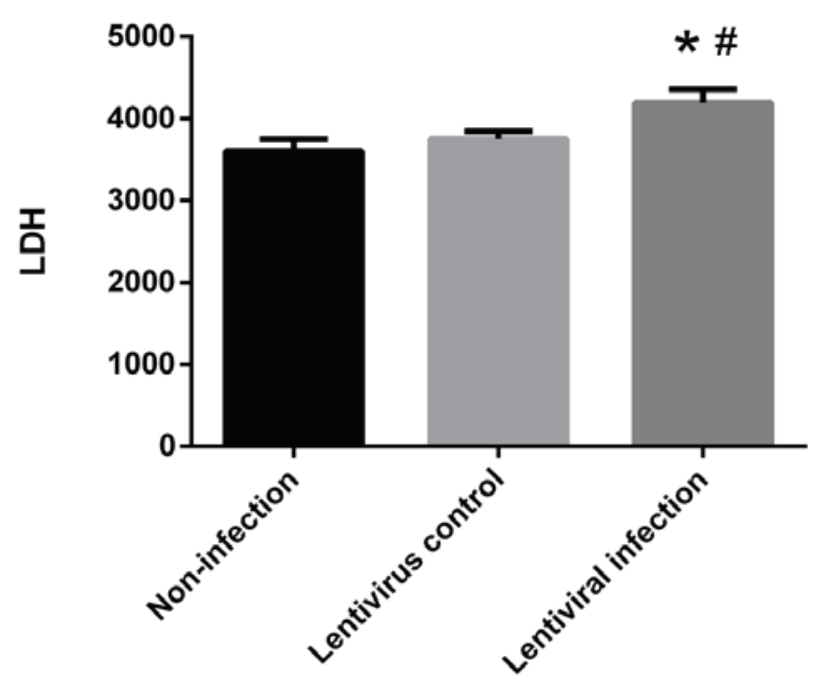

Figure 7. HSP70 silencing promotes the release of LDH. Following A549 cell exposure to hypoxia/reoxygenation for 6 and $24 \mathrm{~h}$ respectively, cell culture medium was collected for $\mathrm{LDH}$ determination. lentivirus control $\left({ }^{\sharp} \mathrm{P}<0.05\right.$ vs. the lentivirus control group; ${ }^{*} \mathrm{P}<0.05$ vs. the non-infection group. HSP70, heat shock protein 70 ; LDH, lactate dehydrogenase.

PI3K/AKT signaling pathways. The results revealed that decreased levels of HSP70 were accompanied with a decrease in p-ERK and p-AKT, indicating that HSP70 is required for ERK and AKT phosphorylation. In addition, the inhibition of ERK and AKT phosphorylation by their respective inhibitors also resulted in a decrease in HSP70 expression. Therefore, the present results indicate that HSP70 may function in an anti-apoptotic manner via the MAPK/ERK and PI3K/AKT signaling pathways.

In conclusion, HSP70 provided protection against hypoxia/reoxygenation. Silencing HSP70 was revealed to decrease cell viability and increase apoptosis in LIRI, which was accompanied by the inhibition of the ERK and AKT signaling pathways. The present study also highlighted the potential role of HSP70 in lung protection. Therefore, it is hypothesized that HSP70 may be a potential target for the treatment of LIRI. Although A549 cells have many features of normal type II alveolar epithelial cells, there are also differences between them (43). Therefore, the in-vivo protective effects of HSP70 require further study.

\section{Acknowledgements}

The authors would like to thank Dr. Bing Luo and Dr. Wen Liu from Qingdao University (Qingdao, China) for their help in the preparation and modification of this manuscript.

\section{Funding}

The present study was supported by grants from the National Natural Science Foundation of China (grant nos. 81571938 and 81501706).

\section{Authors' contributions}

CZ performed the experiments, collected and analyzed the data, and prepared the manuscript. JL collected the data. KW analyzed the data. QL conceived and designed the study, and performed the literature search. YQ conceived and designed the study, and reviewed manuscript.

\section{Availability of data and materials}

All data generated or analyzed during this study are included in this published article.

\section{Ethics approval and consent to participate}

Not applicable.

\section{Patient consent for publication}

Not applicable.

\section{Competing interests}

The authors declare that they have no competing interests. 
A

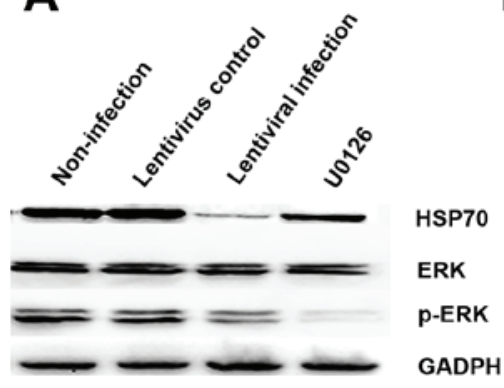

B

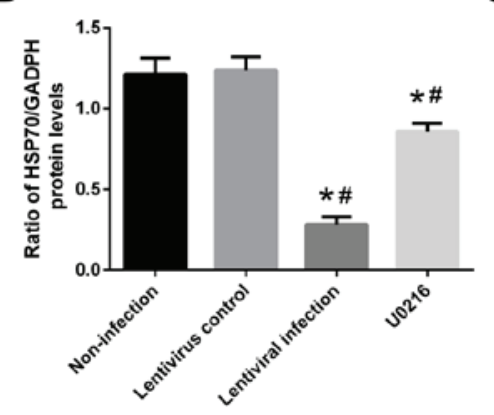

C

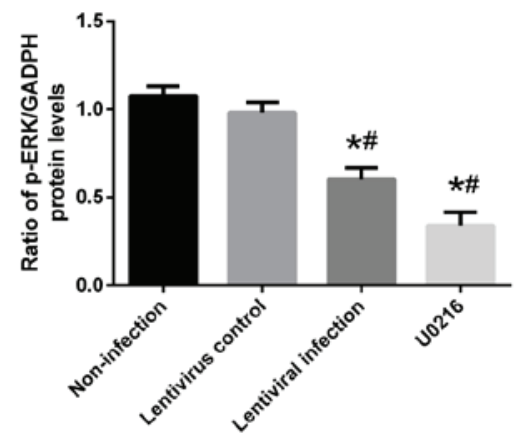

Figure 8. Effect of HSP70 silencing on the mitogen-activated protein kinase/ERK signaling pathways. A549 cells were exposed to hypoxia for $6 \mathrm{~h}$ with or without $10 \mu \mathrm{M}$ U0126. (A) Western blotting was performed to determine the protein expression of HSP70, p-ERK and total ERK. GADPH was used as the loading control. Following western blotting, the ratios of (B) HSP70/GAPDH and (C) p-ERK\ERK were calculated. "P $<0.05$ vs. the non-infection group; ${ }^{\#} \mathrm{P}<0.05$ vs. the lentivirus control group. HSP70, heat shock protein 70; MAPK, mitogen-activated protein kinase; ERK, extracellular signal-regulated kinase; p-, phosphorylated.

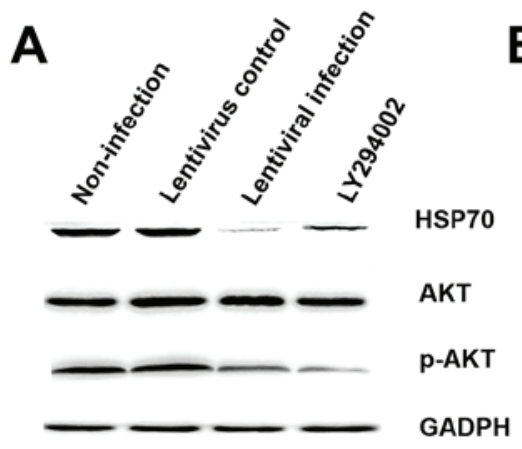

B

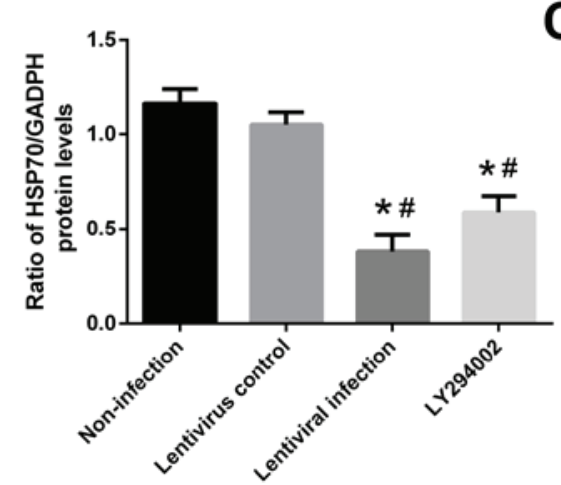

c

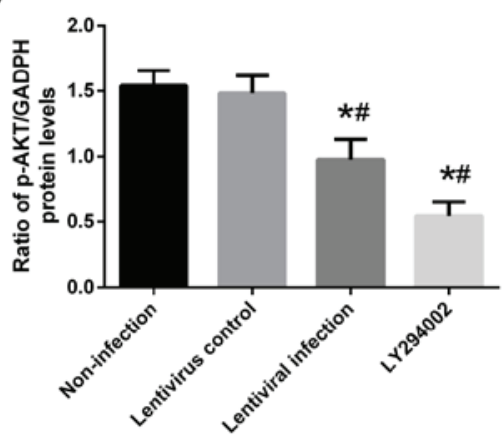

Figure 9. Effect of HSP70 silencing on the PI3K/AKT signaling pathway. A549 cells were exposed to hypoxia for $6 \mathrm{~h}$ with or without $50 \mu \mathrm{M}$ LY294002. (A) Western blotting was performed to determine the protein expressions of HSP70, p-AKT and total AKT. GADPH was used as the loading control. Following western blotting, the ratios of (B) HSP70/GAPDH and (C) p-AKT $\backslash$ total-AKT were calculated. ${ }^{~} \mathrm{P}<0.05$ vs. the lentivirus control group; ${ }^{*} \mathrm{P}<0.05$ vs. the non-infection group. HSP70, heat shock protein 70; PI3K, phosphoinositide 3-kinase; AKT, protein kinase B; p-, phosphorylated.

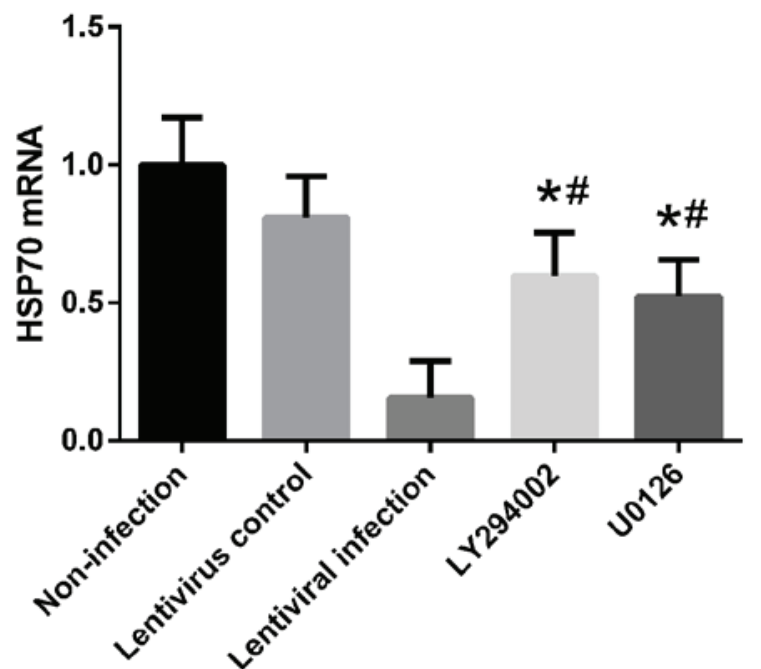

Figure 10. Inhibition of the AKT and ERK signaling pathway results in the decreased expression of HSP70 mRNA. Following A549 cell exposure to hypoxia for $6 \mathrm{~h}$ with or without $10 \mu \mathrm{M} \mathrm{U} 0126$ or $50 \mu \mathrm{M} \mathrm{LY} 294002$, the mRNA levels of HSP70 were detected via reverse transcription-quantitative polymerase chain reaction. ${ }^{~} \mathrm{P}<0.05$ vs. the lentivirus control group; ${ }^{*} \mathrm{P}<0.05$ vs. the non-infection group. AKT, protein kinase B; ERK, extracellular signal-regulated kinase; HSP70, heat shock protein 70.

\section{References}

1. de Perrot M, Liu M, Waddell TK and Keshavjee S: Ischemia-reperfusion-induced lung injury. Am J Respir Crit Care Med 167: 490-511, 2003.

2. Cottini SR, Lerch N, de Perrot M, Treggiari MM, Spiliopoulos A, Nicod L and Ricou B: Risk factors for reperfusion injury after lung transplantation. Intensive Care Med 32: $557-563,2006$.

3. Shimamoto A, Pohlman TH, Shomura S, Tarukawa T, Takao M and Shimpo H: Toll-like receptor 4 mediates lung ischemia-reperfusion injury. Ann Thorac Surg 82: 2017-2023, 2006.

4. Ambrosio G and Tritto I: Reperfusion injury: Experimental evidence and clinical implications. Am Heart J 138: S69-S75, 1999.

5. Quadri SM, Segall L, de Perrot M, Han B, Edwards V, Jones N, Waddell TK, Liu M and Keshavjee S: Caspase inhibition improves ischemia-reperfusion injury after lung transplantation. Am J Transplant 5: 292-299, 2005.

6. Fischer S, Maclean AA, Liu M, Cardella JA, Slutsky AS, Suga M, Moreira JF and Keshavjee S: Dynamic changes in apoptotic and necrotic cell death correlate with severity of ischemia-reperfusion injury in lung transplantation. Am J Respir Crit Care Med 162: 1932-1939, 2000.

7. Radons J: The human HSP70 family of chaperones: Where do we stand? Cell Stress Chaperones 21: 379-404, 2016.

8. van Eden W, van der Zee R and Prakken B: Heat-shock proteins induce T-cell regulation of chronic inflammation. Nat Rev Immunol 5: 318-330, 2005. 
9. Mayer MP and Bukau B: Hsp70 chaperones: Cellular functions and molecular mechanism. Cell Mol Life Sci 62: 670-684, 2005.

10. Gao Y, Han C, Huang H, Xin Y, Xu Y, Luo L and Yin Z: Heat shock protein 70 together with its co-chaperone CHIP inhibits TNF-alpha induced apoptosis by promoting proteasomal degradation of apoptosis signal-regulating kinase1. Apoptosis 15: 822-833, 2010.

11. Liu S, Xu J, Fang C, Shi C, Zhang X, Yu B and Yin Y: Over-expression of heat shock protein 70 protects mice against lung ischemia/reperfusion injury through SIRT1/AMPK/eNOS pathway. Am J Transl Res 8: 4394-4404, 2016.

12. Paul S and Mahanta S: Association of heat-shock proteins in various neurodegenerative disorders: Is it a master key to open the therapeutic door? Mol Cell Biochem 386: 45-61, 2014.

13. Yamashima T: Hsp70.1 and related lysosomal factors for necrotic neuronal death. J Neurochem 120: 477-494, 2012.

14. Hiratsuka M, Mora BN, Yano M, Mohanakumar T and Patterson GA: Gene transfer of heat shock protein 70 protects lung grafts from ischemia-reperfusion injury. Ann Thorac Surg 67: 1421-1427, 1999.

15. Li J, Zhang Y, Li C, Xie J, Liu Y, Zhu W, Zhang X, Jiang S, Liu L and Ding Z: HSPA12B attenuates cardiac dysfunction and remodelling after myocardial infarction through an eNOS-dependent mechanism. Cardiovasc Res 99: 674-684, 2013.

16. Chi W, Meng F, Li Y, Wang Q, Wang G, Han S, Wang P and Li J: Downregulation of miRNA-134 protects neural cells against ischemic injury in N2A cells and mouse brain with ischemic stroke by targeting HSPA12B. Neuroscience 277: 111-122, 2014

17. Jayanthi S, Deng X, Bordelon M, McCoy MT and Cadet JL: Methamphetamine causes differential regulation of pro-death and anti-death $\mathrm{Bcl}-2$ genes in the mouse neocortex. FASEB J 15: 1745-1752, 2001.

18. Nuñez G, Benedict MA, Hu Y and Inohara N: Caspases: The proteases of the apoptotic pathway. Oncogene 17: 3237-3245, 1998.

19. Wu H, Che X, Zheng Q, Wu A, Pan K, Shao A, Wu Q, Zhang J and Hong Y: Caspases: A molecular switch node in the crosstalk between autophagy and apoptosis. Int J Biol Sci 10 1072-1083, 2014.

20. Qi Z, Qi S, Gui L, Shen L and Feng Z: Daphnetin protects oxidative stress-induced neuronal apoptosis via regulation of MAPK signaling and HSP70 expression. Oncol Lett 12: 1959-1964, 2016.

21. Zhang L, Ding W, Sun H, Zhou Q, Huang J, Li X, Xie Y and Chen J: Salidroside protects PC12 cells from $\mathrm{MPP}^{+}$-induced apoptosis via activation of the PI3K/Akt pathway. Food Chem Toxicol 50: 2591-2597, 2012.

22. Shoji T, Yoshida S, Mitsunari M, Miyake N, Tsukihara S, Iwabe T, Harada T and Terakawa N: Involvement of p38 MAP kinase in lipopolysaccharide-induced production of pro- and anti-inflammatory cytokines and prostaglandin $\mathrm{E}(2)$ in human choriodecidua. J Reprod Immunol 75: 82-90, 2007.

23. López-Neblina F and Toledo-Pereyra LH: Phosphoregulation of signal transduction pathways in ischemia and reperfusion. J Surg Res 134: 292-299, 2006.

24. Kamiya T, Kwon AH, Kanemaki T, Matsui Y, Uetsuji S, Okumura T and Kamiyama Y: A simplified model of hypoxic injury in primary cultured rat hepatocytes. In Vitro Cell Dev Biol Anim 34: 131-137, 1998.

25. Livak KJ and Schmittgen TD: Analysis of relative gene expression data using real-time quantitative PCR and the 2(-Delta Delta C(T)) method. Methods 25: 402-408, 2001.

26. Ng CS, Wan S, Arifi AA and Yim AP: Inflammatory response to pulmonary ischemia-reperfusion injury. Surg Today 36 : 205-214, 2006.

27. Ng CS, Wan S and Yim AP: Pulmonary ischaemia-reperfusion injury: Role of apoptosis. Eur Respir J 25: 356-363, 2005.
28. Antoniou KM, Pataka A, Bouros D and Siafakas NM: Pathogenetic pathways and novel pharmacotherapeutic targets in idiopathic pulmonary fibrosis. Pulm Pharmacol Ther 20: 453-461, 2007.

29. Giffard RG and Yenari MA: Many mechanisms for hsp70 protection from cerebral ischemia. J Neurosurg Anesthesiol 16: 53-61, 2004.

30. Kato K, Yamanaka K, Hasegawa A and Okada S Dimethylarsinic acid exposure causes accumulation of Hsp72 in cell nuclei and suppresses apoptosis in human alveolar cultured (L-132) cells. Biol Pharm Bull 22: 1185-1188, 1999.

31. Wang H, Dong Y and Cai Y: Alanyl-glutamine prophylactically protects against lipopolysaccharide-induced acute lung injury by enhancing the expression of HSP70. Mol Med Rep 16: 2807-2813, 2017.

32. Higashi T, Takechi H, Uemura Y, Kikuchi H and Nagata K Differential induction of mRNA species encoding several classes of stress proteins following focal cerebral ischemia in rats. Brain Res 650: 239-248, 1994

33. Zhang H, Okamoto M, Panzhinskiy E, Zawada WM and Das M: PKCס/midkine pathway drives hypoxia-induced proliferation and differentiation of human lung epithelial cells. Am J Physiol Cell Physiol 306: C648-C658, 2014.

34. Hu Z, Huang J, Li Q, Yang J, Zhong L and Zeng Q: Effect of hypoxia on infiltration and migration of lung cancer cells and expression of MMP-2 and TIMP-2. Zhongguo Fei Ai Za Zhi 8: 270-273, 2005 (In Chinese).

35. Pietenpol JA and Stewart ZA: Cell cycle checkpoint signaling: Cell cycle arrest versus apoptosis. Toxicology 181-182: 475-481, 2002.

36. Tanoue T and Nishida E: Molecular recognitions in the MAP kinase cascades. Cell Signal 15: 455-462, 2003.

37. Johnson GL and Lapadat R: Mitogen-activated protein kinase pathways mediated by ERK, JNK, and p38 protein kinases. Science 298: 1911-1912, 2002

38. Chao X, Zao J, Xiao-Yi G, Li-Jun M and Tao S: Blocking of PI3K/AKT induces apoptosis by its effect on NF- $\kappa \mathrm{B}$ activity in gastric carcinoma cell line SGC7901. Biomed Pharmacother 64: 600-604, 2010.

39. Plotkin LI and Bellido T: Bisphosphonate-induced, hemichannel-mediated, anti-apoptosis through the Src/ERK pathway: A gap junction-independent action of connexin43. Cell Commun Adhes 8: 377-382, 2001.

40. Bezler M, Hengstler JG and Ullrich A: Inhibition of doxorubicin-induced HER3-PI3K-AKT signalling enhances apoptosis of ovarian cancer cells. Mol Oncol 6: 516-529, 2012.

41. Qi Z, Qi S, Gui L, Shen L and Feng Z: Daphnetin protects oxidative stress-induced neuronal apoptosis via regulation of MAPK signaling and HSP70 expression. Oncol Lett 12: 1959-1964, 2016

42. Ji K, Xue L, Cheng J and Bai Y: Preconditioning of H2S inhalation protects against cerebral ischemia/reperfusion injury by induction of HSP70 through PI3K/Akt/Nrf2 pathway. Brain Res Bull 121: 68-74, 2016.

43. Oraldi M, Saracino S, Maggiora M, Chiaravalloti A, Buemi C, Martinasso G, Paiuzzi E, Thompson D, Vasiliou V and Canuto RA: Importance of inverse correlation between ALDH3A1 and PPAR $\gamma$ in tumor cells and tissue regeneration. Chem Biol Interact 191: 171-176, 2011.

This work is licensed under a Creative Commons Attribution-NonCommercial-NoDerivatives 4.0 International (CC BY-NC-ND 4.0) License. 